\title{
MILO - A Proposal of Multiple Intelligences Learning Objects
}

\author{
Hamilton de Matos, Pollyana Notargiacomo Mustaro and \\ Ismar Frango Silveira \\ Mackenzie Presbyterian University, São Paulo, Brazil
}

\author{
hamilton.matos@accenture.com, polly@mackenzie.br \\ ismar@mackenzie.br
}

\begin{abstract}
Learning objects-driven approaches for the development of instructional content have been widely used to structure entire courses and repositories for distinct learning contexts. Nonetheless, their use is still done in a static, non-adaptive manner, since students are presented to prebuilt compilations of learning object having few or none relationship with its learning current conditions, history or personal learning style, which together compose the student momentum.
\end{abstract}

This work presents an analysis of current instructional design and multiple intelligences theories in order to create learning objects that provide adaptive learning methods according to different students' characteristics. Using technologies that allow such dynamic approaches, it was created as a proof of concept - a learning object about the Pythagorean Theorem.

Keywords: Learning Objects, Instructional Design, Multiple Intelligences, RIA, Design Patterns.

\section{Introduction}

The current educational paradigm requires a review on the development processes for elaborating learning objects. The differences between students are, most of the times, not taken into consideration, leading to the creation of inefficient resources which do not provide an environment for the students to engage and exercise their potentialities. In order to elaborate a learning object in which the student is the main focus it is necessary to use appropriate technological tools as well as theories which provide ways for creating efficient and customized approaches.

This research aimed to analyze recent instructional design theories, and, supported by multiple intelligences and learning styles theories, to elaborate an effective implementation of a learning object through technologies which allow dynamic, adaptive approaches.

Instructional design proposals deal with a huge amount of variables like systemic structure. It implies in producing data analysis in order

Material published as part of this publication, either on-line or in print, is copyrighted by the Informing Science Institute. Permission to make digital or paper copy of part or all of these works for personal or classroom use is granted without fee provided that the copies are not made or distributed for profit or commercial advantage AND that copies 1) bear this notice in full and 2) give the full citation on the first page. It is permissible to abstract these works so long as credit is given. To copy in all other cases or to republish or to post on a server or to redistribute to lists requires specific permission and payment of a fee. Contact Publisher@InformingScience.org to request redistribution permission. to provide student with situations which are relevant for his needs and peculiarities. Besides, instructional design structures deal with probabilities, which significantly improve learning efficiency, but do not cope with all possibilities involved in this process. However, this problem can be mitigated through an ongoing evaluation of the learner performance. In this way, alternatives and 
different approaches for a specific content to be learned should be presented to the learner throughout the use of the learning object.

The organization of this research is described as follows: section Learning Objects: instructional design and multiple intelligences approaches presents concepts related to learning objects, instructional design and multiple intelligences theories; section Modeling Learning Objects of Dynamic Media Using Design Patterns deals with the architecture proposed for building the application as well as the technologies used; section Developing a MILO shows the storyboard structure applied and the results collected; and, finally, last section presents Conclusions and Further Works.

\section{Learning Objects: Instructional Design and Multiple Intelligences Approaches}

Lowerison et al. (2003) define learning objects as any type of object which facilitates learning in a media context based on computer. Wiley (2002) broadens the definition, considering learning object any digital device which could be used to support learning.

Reigeluth and Nelson (1997) suggest that instructional materials should be divided in small reusable parts. Once teachers grant access to these parts, it is possible to rearrange the structure making it possible to reach the goals of an instruction or educational process. It is possible to follow such guidelines while building learning objects by creating independent instructions or educational resources which might, later, be used together.

Such learning objects can be used for different purposes as well as for distinct kinds of training. Some e-learning proposals which use such objects are listed below (Chairman, n.d.):

- $\quad C B T$ (Computer Based Training) - CBTs are training software where computer plays the role of an instructor. Activities are presented to learners through tests, simulations and exercises, among other activities. CBTs are usually delivered through CD-ROMs and intranets.

- $\quad W B T$ (Web Based Training) - A CBT which is accessed over the internet is considered a WBT. In this kind of training the materials are available in Web servers, which allow its use by multiple learners simultaneously.

- ILT (Instructor Lead Training) - In this kind of training the instructor is the person who guides the learners group during the learning process.

The development, implementation and availability of learning objects for e-learning proposals are made through LMS (Learning Management Systems) or through LCMS (Learning Content Management Systems). LMS allow the distribution of learning contents to students, with resources like visualizing the progress and performance during the use of the tool, for example. LCMS, in a complementary way, provide resources to control the degree of granularity of the learning object to structures them dynamically according to learning styles, for example.

Such systems use a standard for making contents available through different contexts, named SCORM (Sharable Content Object Reference Model), which was developed by ADL (Advanced Distributed Learning). It started as part of the activities of the American Defense Department, but, later, it became part of researches of many international partners and academic institutions (SCORM, 2006). There are some other industry-aware standards, like IEEE LOM (Learning Objects Metadata) (IEEE LTSC, 2005) but in the scope of this work, only SCORM will be considered. 
According to Mackenzie (2004), the function of SCORM is managing the way how a specific content should be inserted in a LMS, how a content should be presented to learners and how the information concerning the progress, in a given context for a content, should be sent to the LMS. The use of SCORM also provides a uniform communication of products created by different teams, like content manager applications developers and content developers.

In this context, it is possible to say that SCORM was developed to promote the integration between different learning objects. The possibility of combining is what makes SCORM a sharable model. Mackenzie (2004) defines the six main objectives which guide SCORM: accessibility, adaptability, production cost saving, durability, interoperability and reusability. In order to develop proper educational materials, such resources and pattern proposals should be used with the support of instructional design theories.

Instructional design aims to analyze the learner needs in order to create educational elements that provide ways to achieve a goal. According to Gagné et al. (1992), an instructional system can be defined as an organization of resources and procedures used to promote learning.

In instructional design theory, Reigeluth (1999) says that apprentices must be at the top of the instructional structure. One consequence of this new paradigm is the fact that some design decisions should be taken by apprentices during their instruction. A learning object based on multiple intelligences theories is in accordance to Reigeluth's ideas, since it places the apprentice correctly during the instruction and also attends the learning needs, once the software provides approaches to distinct learning styles. Instructional design theory offers, therefore, clear guidelines regarding the way people can develop themselves and learn in better a way.

Another characteristic present in instructional design theories is the specification of instructional methods and situations where such methods should be applied or not, so allowing, during the development stage, their separation and/or splitting into more detailed components. This increases the probability of attaining a given goal and also provides further guidelines to the educators. Although this approach helps increasing the possibilities of reaching an objective, it does not guarantee that it will always be successful all the times it will be applied, due to the fact that the methods are probabilistic and not deterministic. An established path defined by learning object agents (responsible for determining which way to follow according to the inputs and performance of the pupils) may not be the best one, but it will facilitate the stages the apprentice must pass through, increasing the possibilities of the given knowledge to be attained in the educational process.

The instructional design theory is not descriptive and/or deterministic. It implies in fact that this approach could not easily foreseen a result after one sequence of events The bases of deterministic theories are the constant observation and experimentation being used to predict effects of sequences of actions or posterior events of current actions.

Design-oriented theories, on the other hand, could be considered prescriptive, since they offer guidelines on how the methods can be used to get the desired results. An example of this technique for solving a mathematics problem, which would be the educational objective to be reached, can be the demonstrating how the problem can be solved by relating it to previous given challenges. Therefore, the objectives of these two types of theories are distinct and require different forms of research.

While the objective of descriptive theories is the validation, design-oriented theories aims to discover which approach, among many methods by which the learning goals can be reached, better facilitates the performance during the use of the instruction. So, the study of the instructional design architecture - composed by methods, tools and requirements - allows educational environments to be improved, in a way to make the learning process more motivating and customized. 
However, Reigeluth (1999) says that not all the aspects in a given context influence the choice of which methods must be used in the instruction. The methods used to facilitate the learning are considered situational since they can be effective in determined situations and may not cause any effect in others. Such instructional situations are divided in two distinct aspects: the desired outcomes and the instructional conditions. These involve the nature of the subject to be studied, the profile of the apprentice, and the characteristics of the learning environment as well as restrictions to the instructional development. According to this, the instructional situations lead to the desired results. These do not include the learning objective, but the necessary levels of effectiveness, efficiency and attractiveness for the instruction. The effectiveness level is used to measure only the way how the learning objective is reached, not evaluating the objective itself. The efficiency is measured by the division of the level of effectiveness by the time or cost of the instruction. The level of attractiveness, in a complementary way, deals with the form how the apprentice interacts and motivates himself with the instruction.

From this context, it is possible to conclude that there are many different ways to present a given content. Varying parameters in the presentation, one gets subtypes of the more general educational strategy which may be more or equally efficient in relation to other strategies depending on the situations where they are used.

This context can be complemented by the adoption of multiple intelligences theory studies. Such theory is the result of psychologist Howard Gardner researches, who has worked for more than 35 years with researches in cognitive development in normal children and in prodigies; collapse of the cognitive skills in brain damage situations; nature of intelligence, creativity and leadership; and the future of professional ethics in a society guided by businesses.

Gardner (1993) states that multiple intelligences theory broadens the concept of intelligence, presenting it as "the ability to solve problems or to elaborate products that are important in a given environment or cultural community". Although the theory of multiple intelligences was elaborated based on the analysis of biological trends, it was also taken into consideration cultural stimuli that are important in the process of solving problems. In this direction, a given ability may appear in different ways in distinct cultures. Below are some characteristics of the seven types of intelligence identified by Gardner in his early works:

- Musical Intelligence - the fact that some individuals are able to play an instrument perfectly but, on the other hand, are incapable to perform simple tasks like recognizing figures, or, even expressing themselves, as examples, are some of the evidences of the independence of musical intelligence. Aspects of this type of intelligence involve the ability to distinct sound patterns as well as rhythms and tones. Musical intelligence is present among instrumentists, teachers, dancers, etc.

- Bodily-kinesthetic intelligence - Control over the body to perform tasks, ability to express oneself through dance or even creating products are characteristics of this intelligence. It is present among actors, dancers, athletes, etc.

- Logical mathematical intelligence - This intelligence as well as the linguistic one are the basis of I.Q. tests. The logical mathematical intelligence is related to recognizing links between distinct data, recognizing patterns as well as the ability of working with abstract symbols. It is well developed among mathematicians, scientists, programmers, etc.

- Linguistic intelligence - This intelligence is responsible for language and its many different forms like reading, writing and poetry, among others. Linguistic intelligence can be found in poets, writers, journalists, etc. 
- Spatial intelligence - The spatial intelligence deals with the ability of visualizing objects through different angles, observing small details, defining routes, recognizing faces and scenes as well as creating images mentally. It is present in sculptors, architects, etc.

- Interpersonal intelligence - This intelligence is related to the ability of communicating, perceiving contrasts in slight mood changes, behaviors, motivations and intentions of other people. Clearly present in political and religious leaders, teachers, therapists, etc.

- Interpersonal intelligence - Guiding oneself according to the analysis of his feelings and emotions is one of the main characteristics of this kind of intelligence. Philosophers and psychiatrists.

By analyzing these descriptions it is possible to notice that a better performance may be achieved according to the kind of instruction applied since it can be related to specific kinds of intelligences in different degrees. It is also possible to notice that the intelligences can be combined and that they provide interdependent elements. During the last years, this complex topic has been the focus of many researches, including educational technology areas.

Currently, there are few case studies involving automatic inference and application of Multiple Intelligences approach in the development of Learning Objects. This paper's next sections aim to show the whole process of building supporting software architecture for modeling Learning Objects in a Multiple Intelligences-aware way.

\section{Modeling Learning Objects of Dynamic Media: Some Software Engineering Considerations}

Nowadays, one of the challenges when using the Internet as an educational tool is the attempt to provide learning experiences with characteristics that are present in traditional desktop environments. A solution to this may be the use of RIAs - Rich Internet Applications, a term coined by Jeremy Allaire in a Macromedia's white paper (2002). The structure of RIAs allows data to be stored on the application server, delivering to the user interface only the relevant data for interaction. Working in an asynchronous way, the application is delivered to the users through the HTTP transfer protocol. One of the characteristics of client-server approaches is the interoperability between different kinds of operational systems, application servers and databases. The great diversity of types of media present in RIAs implementations, leads browsers and devices vendors to keep improving their products. Aspects like navigability, usability and content management are some of the aspects which have been constantly improved.

The development of RIAs involves three elements: rich client technology, server technology and development tools. The first one makes it possible to the user - through elements like Javascript, Macromedia Player Flash, Java Applets and ActiveX - to render and manipulate the data retrieved from the server. The second one is responsible for integrating the client to the logic and application data. Finally, the development tools are used to build RIA applications. Some of the examples are Macromedia MX, Laszlo, Adobe XDP and Nexaweb.

This RIA structure was also built based on client-server technology using ColdFusion MX 7 for integrating the presentation tier (Adobe/Macromedia Flash Player) to other tiers, and MySQL for databases.

Figure 1 presents the structure of a RIA application based on the technologies previously described. The client receives the application as a Macromedia Player Flash standard file making it possible to the application to communicate with the server and load only specific content and perform tasks without having to load the entire web page every time data is retrieved. The compo- 
nents and ColdFusion pages in the server provide the application logic as well as the communication with the database.

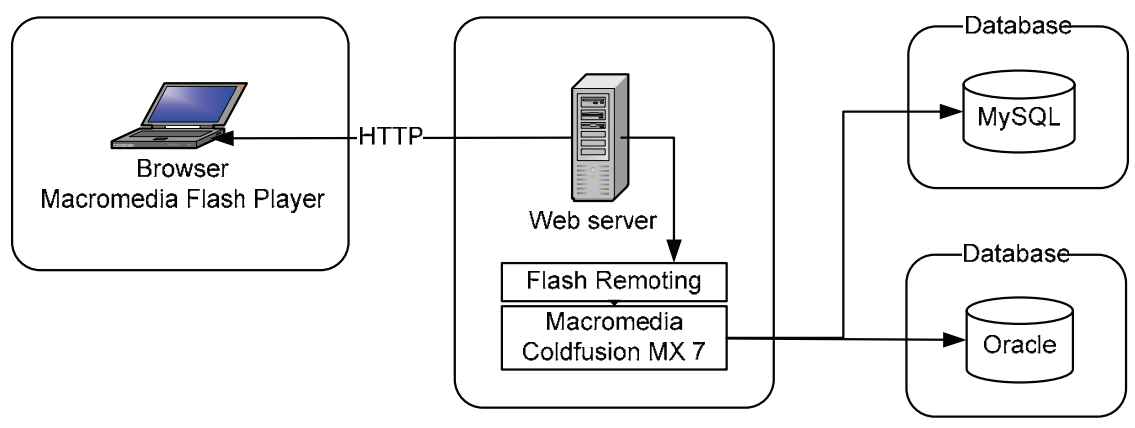

Figure 1 - Structure of an application with RIA and ColdFusion MX7

In the server there are scripts that define the application logic which were created using CFML (ColdFusion Markup Language). In a complementary way, the development of this architecture also involved the study of GoF design patterns (Gamma et al., 1995).

Since technologies like ColdFusion were used, which are based on J2EE environments, the application of the GoF and J2EE design patterns provided a clear and efficient modeling through the use of UML. Fowler (2004) identifies the main advantage in the use of UML notations as the fact that it makes it possible to communicate ideas in a clear and straightforward way, also promoting standardization in the vocabulary used by software developers.

In the definition of the prototype, it was used a five-tiered architecture (Alur et al., 2003). This approach was adopted when building learning objects in order to benefit from some inherent advantages such as easy maintenance, high scalability and reuse, among others. In order to better clarify the underlying aspects of such implementation, some important details related to the Design Patterns-based Software Engineering Modeling process are to be shown in next paragraphs.

The presentation tier (partially presented in Figure 2) is based on an interface that makes a requisition. This is handled by applying the Front Controller J2EE pattern (Alur et al., 2003), which provides a centralized access point for solicitations made to the presentation tier, allowing the maintenance of different types of control, including services like authentication, delegation for processing business rules, use of appropriate visualizations, exception handling and control of strategies for creating contents. An instance of FrontController class communicates with LearningObjectView which, through the LearningobjectViewHelper class, implements the View Helper J2EE pattern (Alur et al., 2003). This encapsulates the business rules providing clients with different visualizations, leading to the dynamic content of the application.

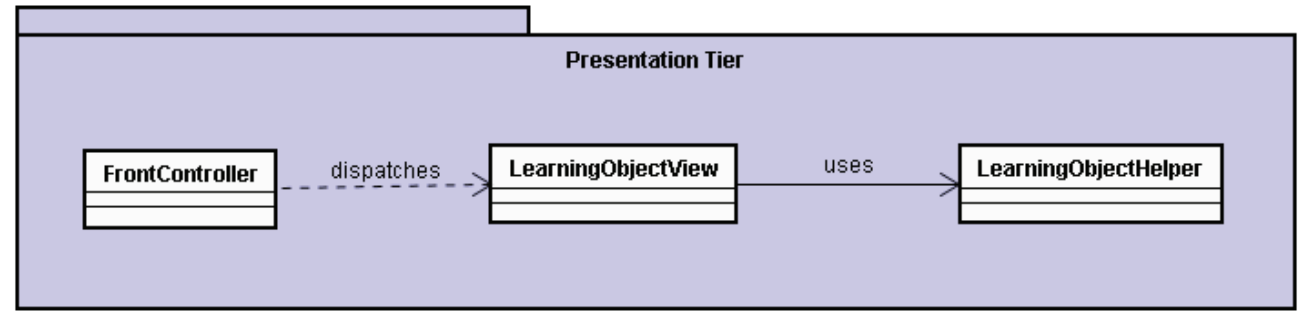

Figure 2 - Presentation tier

Factory Method GoF pattern was first used (Gamma et al., 1995) for creating the strategy to be presented to the apprentice during the use of the learning object, being activated by LearningObjectHelper. Based on the initial pre-test results, used to define the best learn- 
ing styles, the ConcreteFactory class creates the proper object based on these characteristics. Figure 3 shows part of the structure of the application of this pattern.

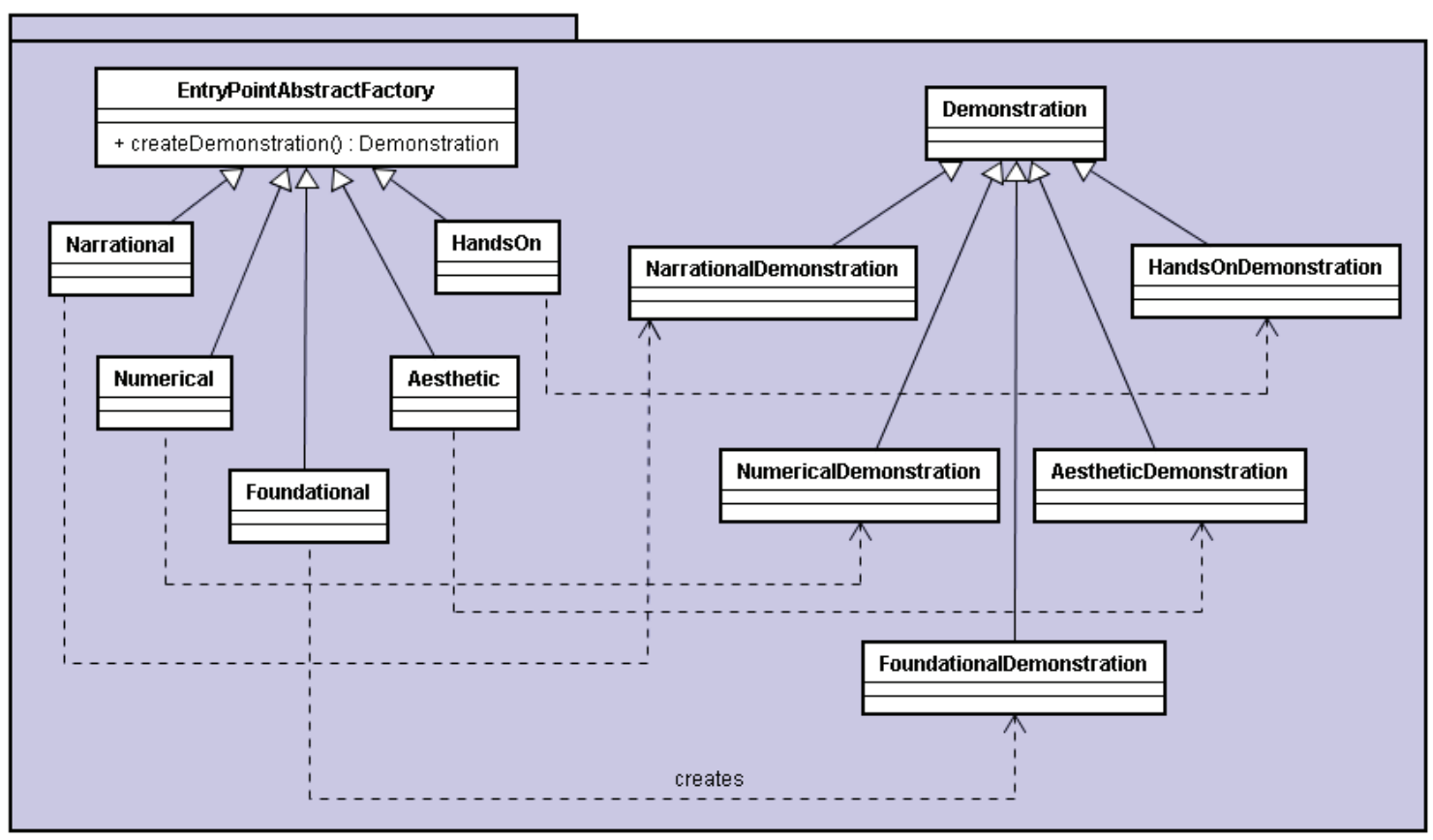

Figure 3 - Creating a learning object through Factory Method pattern

In fact, EntryPointAbstractFactory class was converted to the Abstract Factory GoF pattern by assigning to it new factory methods for creating games, simulations and Socratic dialogues - respectively with the methods createGame (), createSimulation() and createSocraticDialog (). Each one of the entry points was created as a concrete class that extends the EntryPoint class, which is responsible for creating specific objects based on the instructions proposed by Reigeluth (1999). Therefore there was a combination between the six types of entry points proposed by Gardner (1983) and Reigeluth's kinds of instructions.

Since the learning object was built based on flexibility and scalability, facts like instructional design theories changes would not be a problem for modifying the learning object. One of the object oriented techniques guidelines is that fact that classes should be closed for modification but open for extension. Taking as examples the classes that represent the entry points, in case a new point is defined, it is possible, in a simple, safe and fast way, to extend the EntryPoint abstract class by adding a new class for the new entry point, without changing existent code.

The description of this architecture is complemented by the implementation of the resource tier, which uses two separate databases in order to facilitate reuse. The LearningobjectDataSource (Figure 4) is responsible for storing data related to the different types of resources used in the instructions presented to the apprentice like, for example, different types of medium and layouts. The PreTestDataSource stores the pre-tests data, which are used to determine which learning approach should be used. 


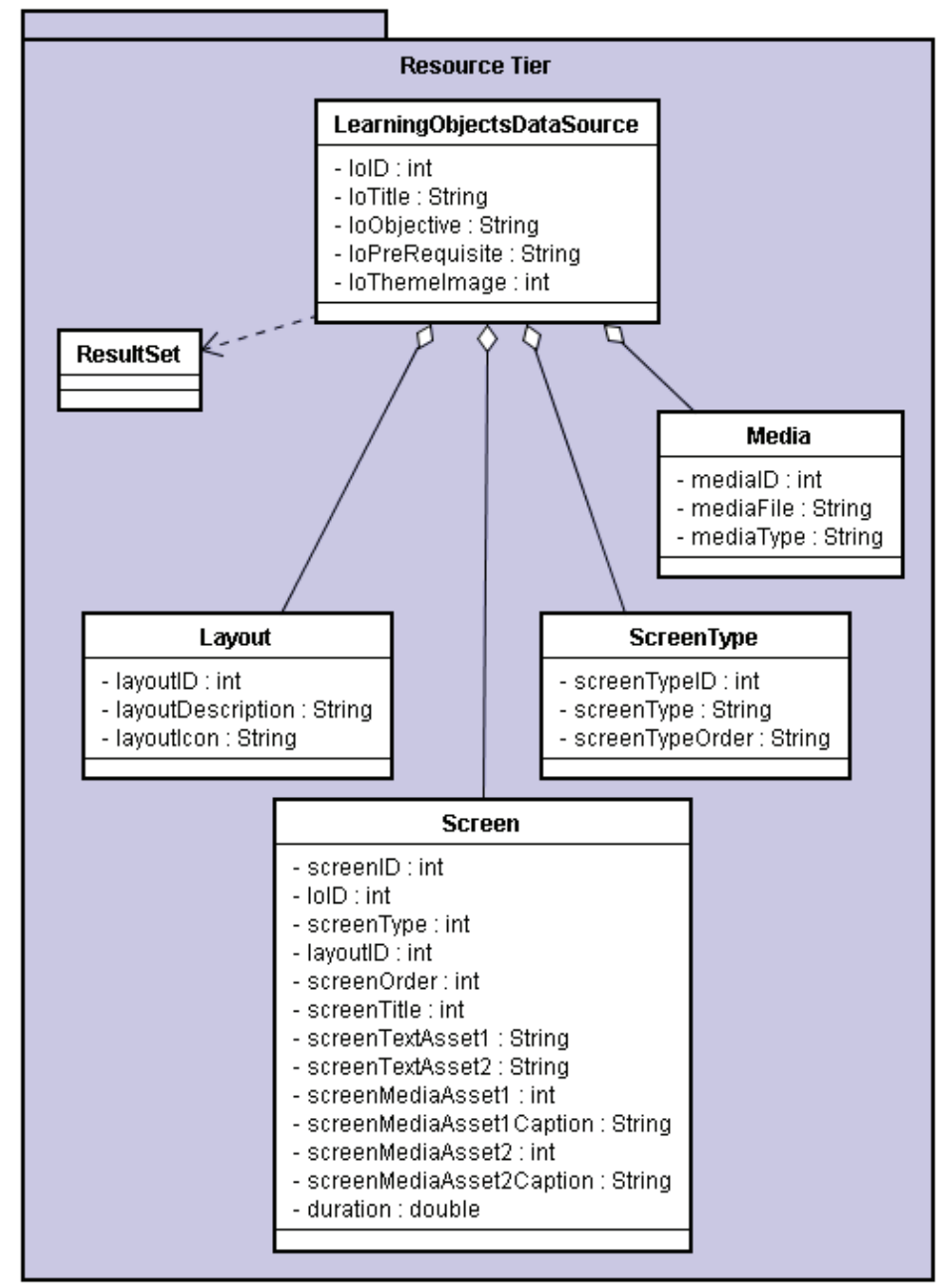

Figure 4 - Resource tier classes

From the concepts previously presented, it is possible to notice that applying design patterns is a relevant element to be considered during the learning object elaboration. According to the architecture here presented, which will be called MILO (Multiple Intelligences Learning Objects), a learning object was developed as a proof of concept.

\section{Developing a MILO}

For the development of a learning object it was taken into consideration the definition of target objectives as well as the enabling objectives (Gagné et al., 1992). In the study of the Pythagorean Theorem, solving a real life problem using such Theorem may be considered a target objective, whereas finding out geometric relations between the elements involved in the problem may be considered the enabling objective.

In combination to these elements, two forms of task analysis were used: procedural and information processing. The first one (Figure 5a) describes the division of the task in stages to be executed by the apprentices so that the final objective is reached. In a complementary form, the second (Figure 5b), which is more detailed, shows the sequence of the instruction and the mapping of the required knowledge or necessary skills for using the object. 


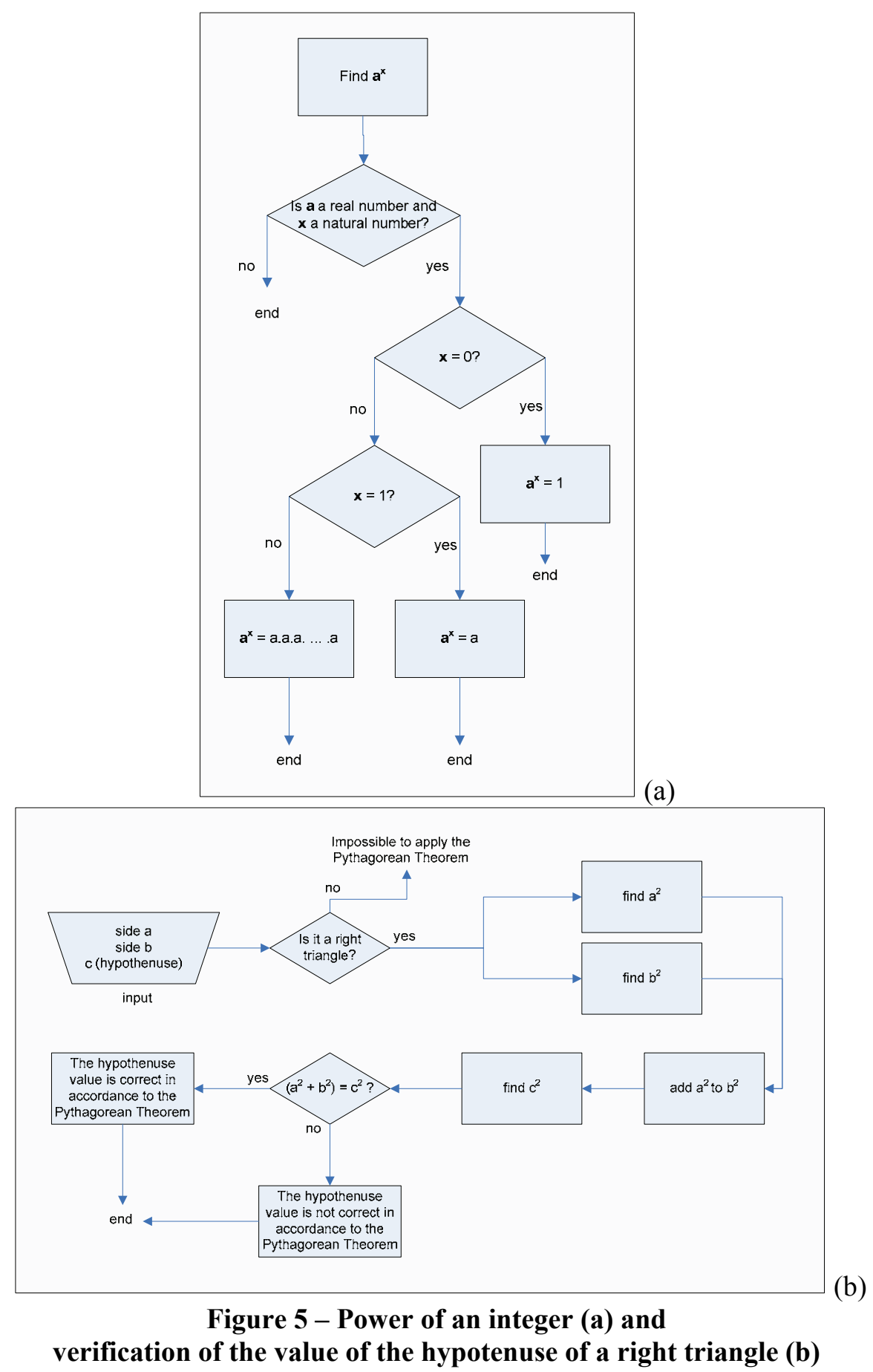

The required skills presented in the diagram become necessary for accomplishing the task. Once these skills are identified, it is possible to define the learning task analysis. This analysis is important for defining the pre-requisites learner must have in order to accomplish the tasks. However, the development of task analysis involves more complex elements organizations as well as taking into consideration the variables related to learning objects techniques used during the development of learning objects.

One manner of keeping a coherent document of learning object organization is the use of storyboards (scripts that describe educational elements, in this case, the learning object). Mustaro et al. 
(2006) define this technique as a visual documentation to represent the architecture, parameters, connections, forms of feedback and other relevant data used to represent the object. Through such educational scripts, it is possible to define themes, instructional goals, pre-requisites (skills and technologies), feedback forms (Figure 6a), types of media, license agreements, level of interactivity, learning strategies, and so on. With these data it is possible to provide the development team with relevant data for taking proper actions as well as which stages should be followed (Figure $6 b)$.

\begin{tabular}{|c|c|c|c|c|c|}
\hline \multicolumn{2}{|l|}{$\begin{array}{l}\text { ID: } 0001 \\
\text { Name: MILO }\end{array}$} & \multicolumn{2}{|c|}{ Difficult Level: Middle } & $\begin{array}{l}\text { Linguistic } \\
\text { Level: Low }\end{array}$ & $\begin{array}{l}\text { Time: } \\
20 \text { minutes }\end{array}$ \\
\hline \multicolumn{2}{|l|}{ Theme } & \multicolumn{4}{|c|}{ Mathematic (high school) } \\
\hline \multicolumn{2}{|c|}{ Learning Goals } & \multicolumn{4}{|c|}{$\begin{array}{l}\text { After learning object use learner will able to concept ab- } \\
\text { straction for real life problems and applies Pythagorean } \\
\text { Theorem to solve them. }\end{array}$} \\
\hline \multicolumn{2}{|l|}{ Objectives } & \multicolumn{4}{|c|}{$\begin{array}{l}\text { Present new approaches that involve diverse mathematic } \\
\text { elements as, for examples, rectangle triangle theory. }\end{array}$} \\
\hline \multicolumn{2}{|c|}{ Tests/Feedback } & \multicolumn{4}{|c|}{$\begin{array}{l}\text { Evaluation is based on tests presented in final of each } \\
\text { module. According to performance is assignment another } \\
\text { instruction to act as reinforcement or development skills. }\end{array}$} \\
\hline \multicolumn{2}{|l|}{ Pre-requisites } & \multicolumn{4}{|c|}{$\begin{array}{l}\text { Apprentices must be able to realize addition, diminution, } \\
\text { and division with click and drag mouse computer. }\end{array}$} \\
\hline \multicolumn{2}{|c|}{ Description/Keywords } & \multicolumn{4}{|c|}{$\begin{array}{l}\text { Present different approaches of Pythagorean Theorem } \\
\text { through use of Instructional Design Theories and Multiple } \\
\text { Intelligences. }\end{array}$} \\
\hline Version: 1 & \multicolumn{2}{|c|}{$\begin{array}{l}\text { Date: } \\
\text { May } 9,2006\end{array}$} & \multicolumn{3}{|c|}{$\begin{array}{l}\text { Comments: It is necessary that browser have } \\
\text { Macromedia Flash Player } 6 \text { or higher installed. }\end{array}$} \\
\hline
\end{tabular}

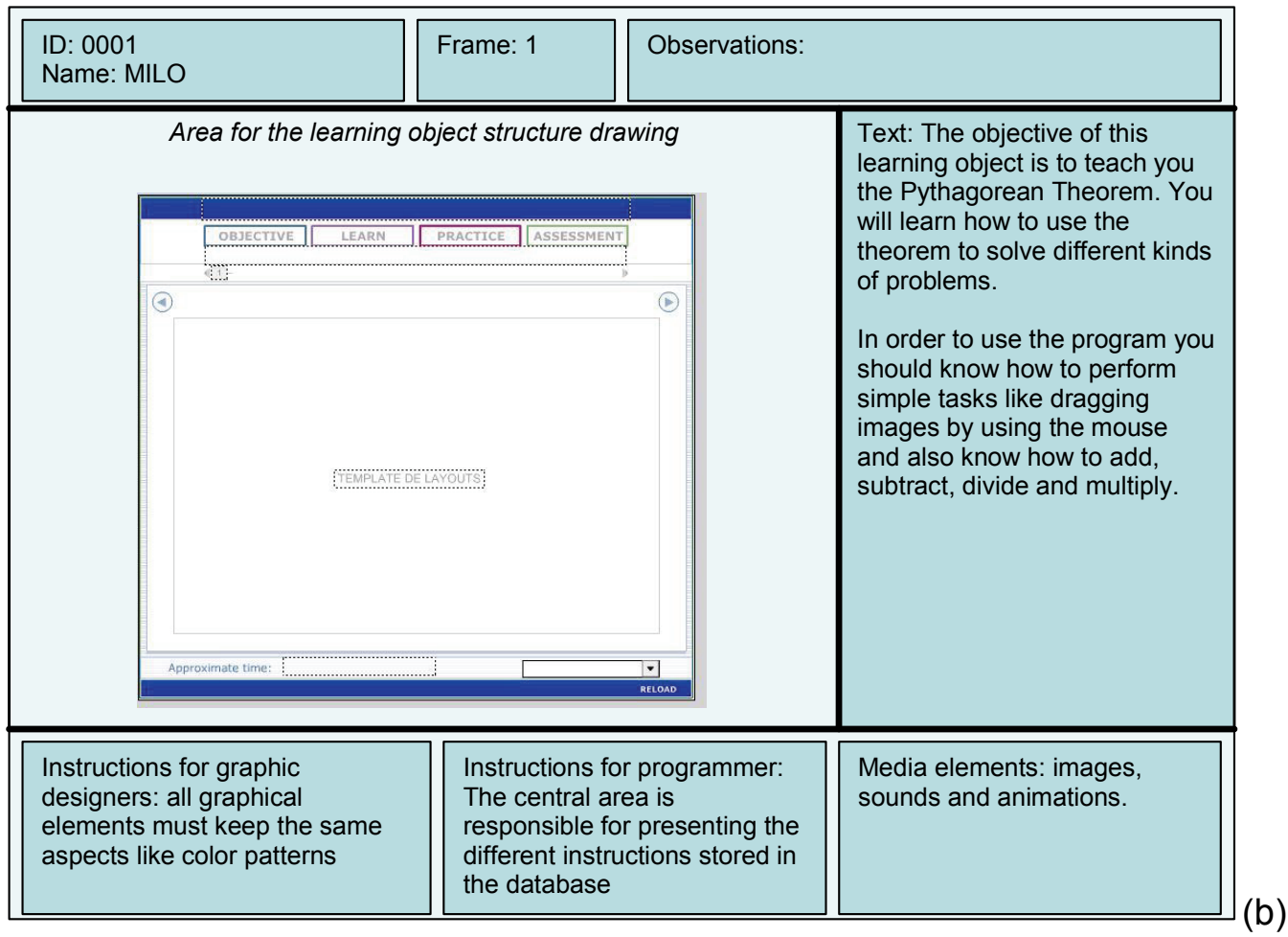

Figure 6 - Document model of educational storyboard for learning object draft 
The study of these elements allowed the definition of the objective of MILO on the Pythagorean Theorem: to work on important concepts concerning this theme, providing the apprentice with experiences involving classic problems on this area. It was also presented situations where the Theorem could be applied as an alternative kind of resolution. From this premise it was established, as prerequisite for using of the learning object, basic knowledge on first degree equations.

Before the apprentice starts the educational work with the Pythagorean Theorem, the object provides a revision of concepts. In this sense, it was attempted to reach learners that already know the subjects related to the prerequisites. It was also attempted to present the content in different forms to pupils who do not have such knowledge or which are not secure while solving problems related to first degree equations. To keep the learner motivation throughout the interaction, it was adopted a strategy to show the importance of the subject. This proposal took the ideas of Ausubel (1978), trying to show a meaning in the content to the apprentice.

One of the goals of the learning object was to involve the learner during the instruction as much as possible, in order to make him active, resulting in a bigger retention of content since aspects like visual activities may increase the efficiency of learning in 89\% (Freeman \& Freeman, 2004). Once the apprentices who will use the learning object are teenagers, it was kept an informal language with a conversational style. Recent studies (Freeman \& Freeman, 2004) show that students had been able to improve $40 \%$ in post learning tests when the tasks had an informal style and also when the tasks spoke directly to the apprentice. These proposals, combined to the studies of Heins \& Himes (2002), leaded to the learning object structure presented in Figure 7, as follows:

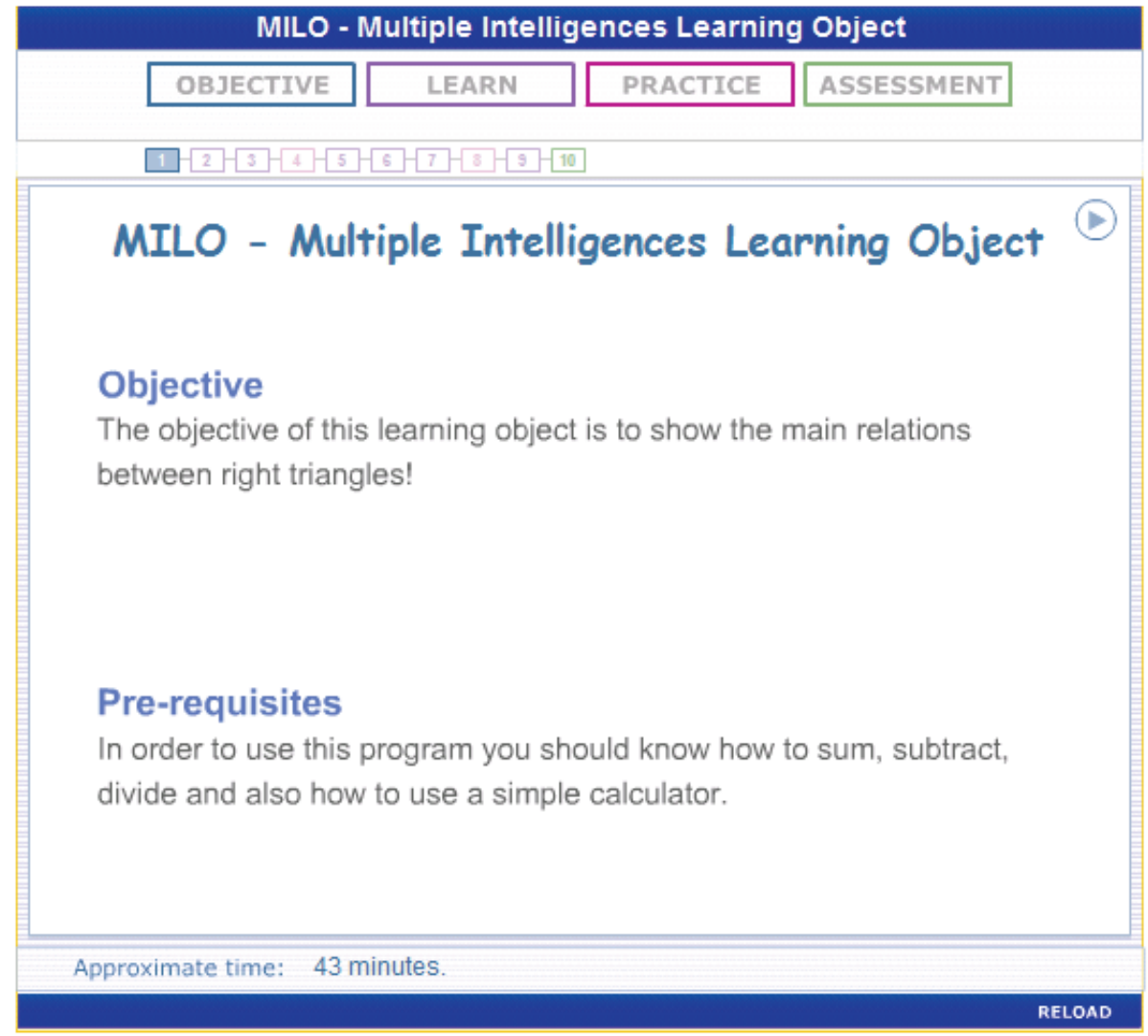

Figure 7 - Structure of the learning object developed 
The central area of the learning object was built to allow the use of many types of layouts like, for example, an animation with a text by the side, an animation only or several other configurations which have as an objective keeping the granularity in the sense that different medium can be used in distinct and independent forms. The learning object was divided in four types of distinct kinds of activities in the upper part of the initial screen: objective, learning, practicing and assessment. With this division it is possible to present the subject in a sequential way or through the choice of one of these tasks. At the moment when one of the four components is chosen, the numbers placed below the four main buttons are activated or inactivated according to the choice. For a better visualization the active numbers have the same color as the corresponding main button.

Through this approach it is possible to add new instructions to the object in a simple form. At the moment when a new instruction is added to the database, it is necessary to enter some keywords. Later these data will be used so that the new instructions can be presented to learners who have characteristics similar to the specifications entered. As examples, figures 8, 9 and 10 presents elements elaborated during this research. In figure 8 the task is to find the angles in the pictures on the left that match to the angles of the pictures on the right.

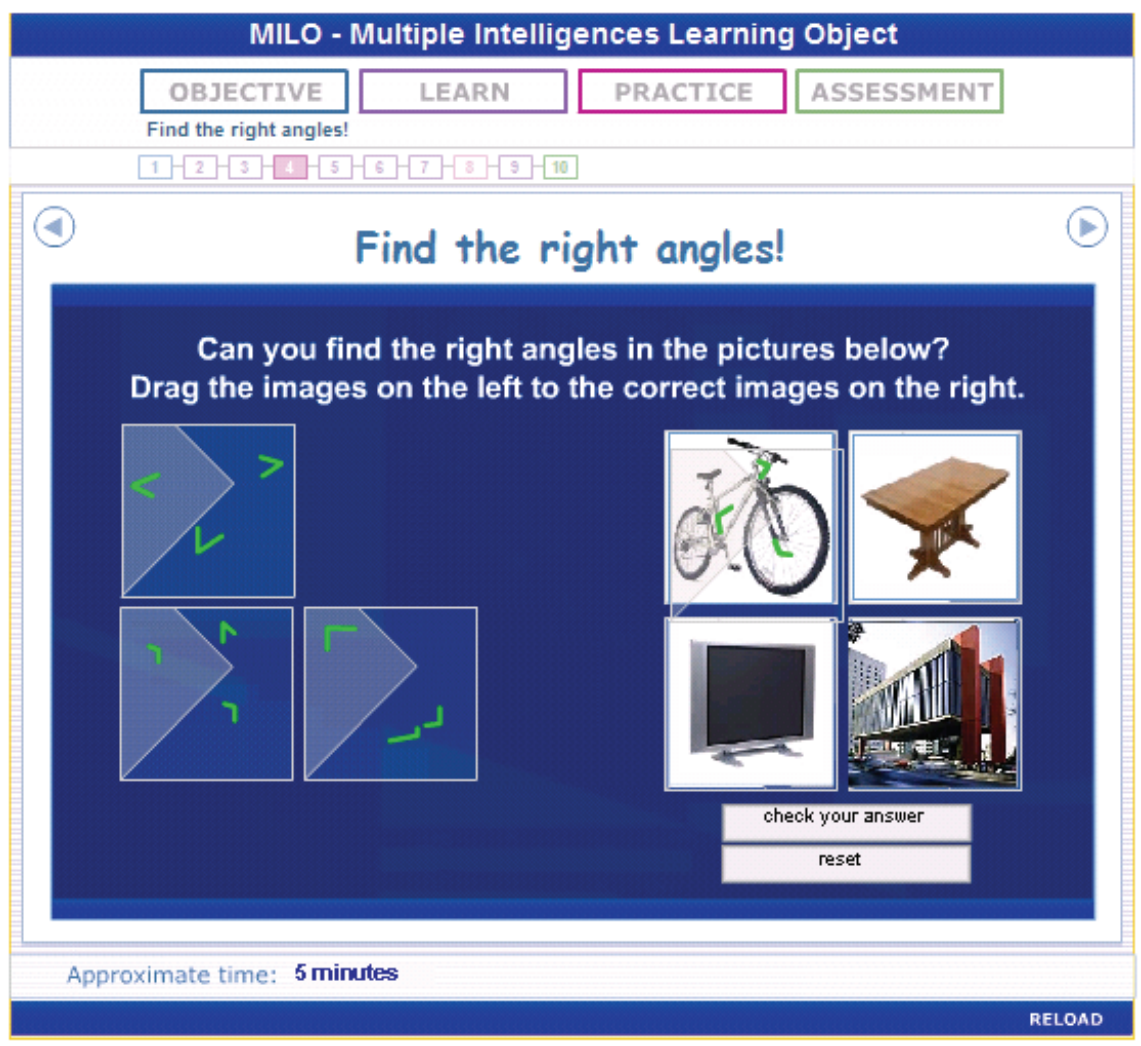

Figure 8 - Game example involving right angles and spatial intelligence

This example shows how an approach using spatial intelligence can be made. There must be an abstraction so that the angles of the images can be visualized as 90 degrees through different perspectives, which are different from the perspectives of the two dimensional images presented. 


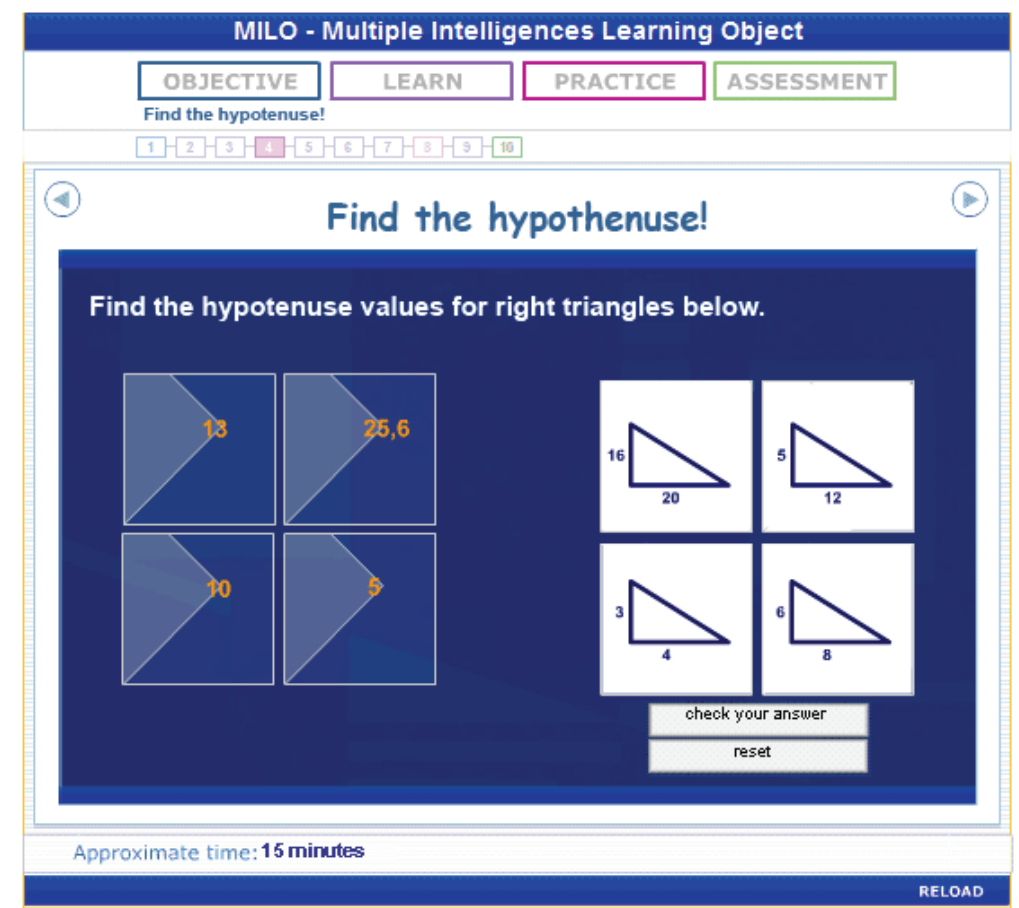
Figure 9 - Exercise involving hypotenuse calculation and logical mathematical
intelligence

This other example presents different triangles with leg numbers. The learners must calculate hypotenuse of each triangle and associate left results with them.

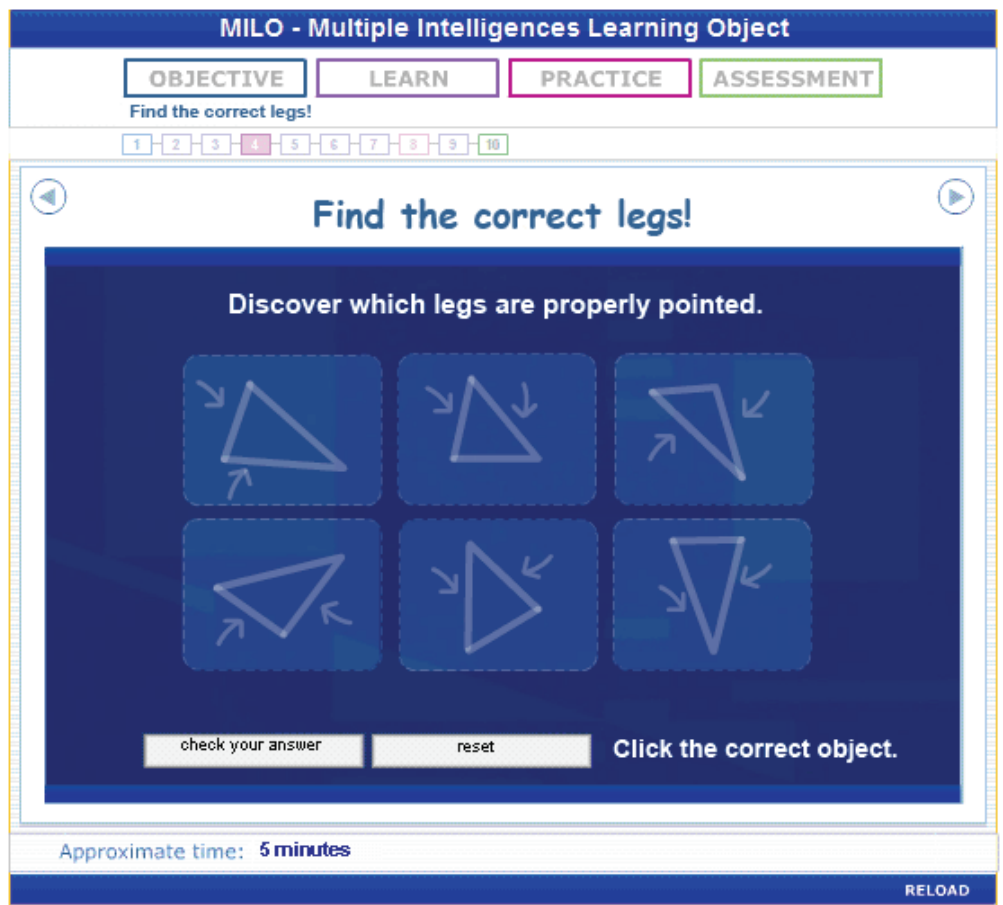

Figure 10 - Example showing legs identification inside a right triangle - spatial intelligence allied to logical-mathematical 
Another example combines spatial and logical-mathematical intelligence when requires to analyze triangles and select elements that indicate legs. In this activity is necessary to think about triangle in rotated spatial perspective and visualize 90 degree angle to identify pointed legs.

\section{Conclusions and Further Work}

A common problem present on most of learning objects nowadays is the fact that they have static and non-customized content. Using current RIA technologies it is possible to create dynamic learning objects to supply the learner needs. In order to create more efficient learning objects it should be taken into consideration that different students learn differently from each other and, also, that one subject can be presented in many different ways, making it possible to deal with multiple intelligences concepts.

It is also important to mention that the use of design patterns with an instructional design methodology was the most important approach used to find the project needs, maintain the stages and document the process of building the learning object. It was possible to find that this approach was also valid to establish an internal coherence between the learning object elements; what it will facilitate, in the future, its improvement, the addition of other elements as well as the use of the same architecture for developing other contents.

From this approach, it was established a basis with learning objects, instructional design and multiple intelligences. It is expected that this research contribute to studies related to the use of current technological resources for implementing learning environments which could better fulfill learners' requirements.

As a future work, it is intended to apply tests to verify the degree of effectiveness and usability of the developed learning object. From these analyses, it will be possible to complement MILO, adding new information so that it can be used as a learning tool in education institutions. Another topic that will be worked is the use of the standard SCORM, what will allow to the insertion of the objects created during this research in a learning object repository, making it possible to make searches through metadata.

\section{References}

Allaire, J. (2002) Macromedia Flash MX - A next-generation rich client. White paper. Retrieved October, 12, 2006 from http://download.macromedia.com/pub/flash/whitepapers/richclient.pdf

Ausubel, D., Novak, J. \& Hanesian, H. (1978). Educational psychology: A cognitive view (2 ${ }^{\text {nd }}$ ed.). Holt, Rinehart \& Winston.

Alur, D., Crupi, J. \& Malks, D. (2003). Core J2EE patterns: Best practices and design strategies (2 ${ }^{\text {nd }}$ ed.). Prentice Hall.

Chairman, V. D. (n.d.) A glossary of e-learning terms and acronyms. The eLearning Network. Retrieved January 20, 2006, from http://www.elearningnetwork.org/articles/article9.doc

Fowler, M. (2004). UML distilled: A brief guide to the standard object modeling language (3rd ed.). Addison-Wesley.

Freeman, E. \& Freeman, E. (2004). Head first design patterns. O’Reilley Media Inc.

Gagné, R. M., Briggs, L. T. \& Wagner, W. W. (1992). Principles of instructional design. Harcount Brace Jovanovich College Publishers.

Gardner, H. E. (1983). Frames of mind: The theory of multiple intelligences. Basic Books.

Gamma, E., Helm, R., Johnson, R. \& Vlissides, J. (1998). Design patterns: Elements of reusable objectoriented software. Addison-Wesley. 
Heins, T. \& Himes, F. (2002). Creating learning objects with Macromedia Flash MX. Adobe. Retrieved January 01, 2006, from http://www.adobe.com/resources/elearning

IEEE LTSC. Learning object metadata. Learning Technology Standards Committee. Retrieved November 20, 2006, from http://ieeeltsc.org/wg12LOM/

Lowerison, G., Gallant, G. \& Boyd, G. (2003). Learning objects in distance education: Addressing issues of quality, learner control and accessibility. Conference Proceedings of CADE-ACED 2003. Retrieved February 08, 2006, from http://www.cadeaced2003.ca/conference_proceedings/Gallant.pdf

Mackenzie, G. (2004). SCORM 2004 primer. A (mostly) painless introduction to SCORM. Retrieved January 20, 2006, from http://www.mcgill.com/media/SCORM_2004_Primer_v1_McGill_Digital Solutions_Gord_Mackenzie.pdf

Mustaro, P. N., Silveira, I. F., Omar, N. \& Stump, S. M. D. (2007). Structure of storyboard for interactive learning objects development. In A. Koohang \& K. Harman (Eds.), Learning objects and instructional design (pp. 253-280). Santa Rosa, California: Informing Science Press.

Perkins, D. N. (1992). Smart schools: Better thinking and learning for every child. The Free Press.

Reigeluth, C. M. \& Nelson, L. M. (1997). A new paradigm of ISD? In R. C. Branch, \& B. B. Minor (Eds.), Educational media and technology yearbook (Vol. 22, pp. 24-35). Libraries Unlimited.

Reigeluth, C. M. (1999). Instructional-design theories and models (Volume II). Lawrence Erlbaum Associates.

SCORM 2004 2nd Edition Documentation. (2006). ADL. Retrieved April 23, 2006, from http://www. adlnet.gov/scorm/history/2004/documents.cfm

Willey, D. A. (2000). Connecting learning objects to instructional design theory: A definition, a metaphor, and a taxonomy. In D. A. Wiley (Ed.), The instructional use of learning objects [Online Version]. Retrieved October 15, 2005, from http://reusability.org/read/chapters/wiley.doc

\section{Biographies}

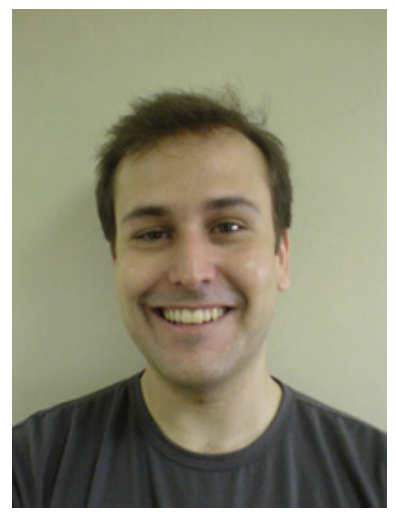

Hamilton de Matos held his BSc. in Computer Science in 2006, at Mackenzie Presbyterian University, located in São Paulo, Brazil. His research field comprises the application of Multiple Intelligences Theory on the development of Learning Objects. Presently he works with software development for Accenture of Brazil.

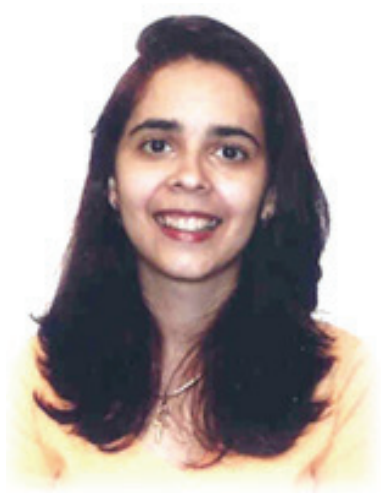

Pollyana Notargiacomo Mustaro obtained her PhD in Education (São Paulo University, 2003), being specialized on Internet hackers' Knowledge Building Process. Nowadays, she is a professor at Mackenzie Presbyterian University. Her research areas include Learning Objects Theory, Learning Styles, Instructional Design, Distance Learning and Hypertext Theory. 


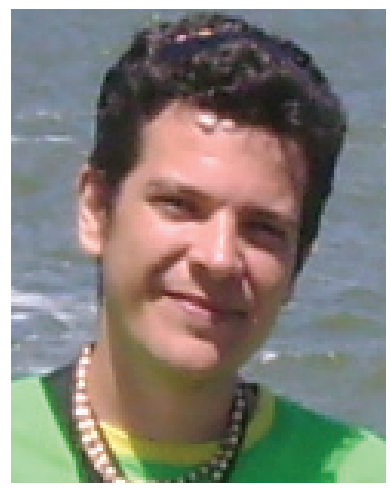

Ismar Frango Silveira defended his $\mathrm{PhD}$ thesis in Distributed Objects applied to Virtual Reality-based Learning Environments in May 2003, at São Paulo University. Currently, he is involved with researches in Adaptive Learning Objects and Collaborative Learning. He presently teaches at Mackenzie Presbyterian University and Cruzeiro do Sul University, both in São Paulo, Brazil. 